\title{
LAS FÓRMULAS RUTINARIAS COMO UNIDADES FRASEOLÓGICAS
}

\author{
M. BELÉn Alvarado OrTEGA \\ Universidad de Alicante. Grupo GRIALE \\ Belen.Alvarado@ua.es
}

\begin{abstract}
Resumen
En este trabajo presentamos un estudio de uno de los fenómenos pragmáticos más difíciles de delimitar, las fórmulas rutinarias. Así, pese a que en los últimos cincuenta años se ha experimentado un notable avance en la caracterización de este tipo de unidades, sigue habiendo una gran diversidad de criterios que dificultan su estudio. Por tanto, es necesario establecer las características de estas unidades fraseológicas y delimitarlas del resto.
\end{abstract}

PALABRAS CLAVE: fórmulas rutinarias, unidades fraseológicas, idiomaticidad.

\begin{abstract}
This article presents an analysis of one of the most controversial phraseological phenomenon, conversational routines. Despite the advances in the characterization of these units in the late fifty years, there is still a great variety of criteria which complicates the study of the mentioned units. Thus, it is necessary to set the main features of phraseological units as well as the field to which it belongs. I propose some characteristics in order to delimit the concept under study and also to differentiate it from other phraseological units.
\end{abstract}

KEYWORDS: conversational routines, phraseological unit, idiomaticity.

\section{Introducción ${ }^{1}$}

En el trabajo que presentamos proponemos definir uno de los tipos más complejos de unidades fraseológicas que existen: las fórmulas rutinarias. Su complejidad se debe a que numerosos autores han hablado de ellas, en general, y de sus rasgos, en particular, pero no han estudiado cuáles son estos. Así, pese a que algunas unidades fraseológicas se encuentran perfectamente delimitadas, como es el caso de los refranes y de las locuciones, otras no han tenido la misma suerte, debido a la dificultad de su funcionamiento, como es el caso de las fórmulas rutinarias (jmadre mía!, iqué bonito!). Por ello, resulta complicado realizar un análisis de fórmulas rutinarias si previamente no se ha estudiado el lugar que ocupan en las unidades fraseológicas. De ahí que propongamos en este artículo deslindar cada una de las propiedades que manifiestan las fórmulas rutinarias y las diferenciemos del resto de unidades fraseológicas (UFs).

\footnotetext{
${ }^{1}$ Grupo GRIALE cuenta con la ayuda del proyecto HUM2004-01318/FILO, concedido por el Ministerio de Educación y Ciencia, que se titula "Análisis pragmático de la ironía verbal. Tipología y aplicaciones a la enseñanza del español como lengua extranjera". Para más información puede consultarse la página web: http://griale.ua.es.
} 
Para realizar este trabajo, nos basaremos en algunos ejemplos de fórmulas rutinarias del español hablado, extraídas del Corpus de conversaciones coloquiales de Briz y el grupo Val.Es.Co. (2002). Gracias a este corpus hemos comprobado la gran aparición que tienen estas UFs en nuestra habla cotidiana, ya que forman parte del acervo lingüístico de los hablantes de español, puesto que nos ayudan a expresar nuestros sentimientos y emociones, $\mathrm{y}$, en ocasiones, permiten desenvolvernos en situaciones de la vida cotidiana.

A continuación, haremos un breve repaso al sustento teórico de las UFs para distinguir las fórmulas rutinarias del resto.

\section{Las unidades fraseológicas}

En las últimas décadas, la fraseología ha sido objeto de estudio de numerosos autores, lo que ha provocado la aparición de diversas obras basadas en este campo. Sin embargo, a pesar de que los estudios sobre este tema han ido en aumento, la variedad terminológica, tanto para referirse a la disciplina general como a los distintos fenómenos y elementos que ésta engloba, pone de manifiesto su inestabilidad.

En la actualidad para hablar del objeto de estudio de la fraseología se utiliza el término unidad fraseológica, ya que está consensuado por los principales investigadores de la materia (Hernando 1990; Corpas 1996; Ruiz Gurillo 1997; Zamora 1998; entre otros), aunque se han utilizado otros términos en español como expresión pluriverbal (Casares 1969 [1950]) o expresión fija (Zuluaga 1980). Corpas (1996: 20), autora de referencia para los principales estudiosos del tema, define a las UFs del siguiente modo:

Son unidades léxicas formadas por más de dos palabras gráficas en su límite inferior, cuyo límite superior se sitúa en el nivel de la oración compuesta. Dichas unidades se caracterizan por su alta frecuencia de uso, y de coaparición de sus elementos integrantes; por su institucionalización, entendida en términos de fijación y especialización semántica; por su idiomaticidad y variación potenciales; así como por el grado en el cual se dan todos estos aspectos en los distintos tipos.

A partir de estas líneas reformulamos una nueva definición de UFs, en la cual entendemos que una UF es cualquier combinación formal y psicolingüísticamente ${ }^{2}$ estable de dos o más palabras con idiomaticidad potencial, que tiene como límite superior la oración compuesta (Alvarado 2008). De esta manera, toda UF debe poder enmarcarse bajo esa definición.

Según el Manual de fraseología española (Corpas 1996: 52), las UFs se engloban en tres esferas:

-Esfera I. Incluye las unidades fraseológicas sólo fijadas en la norma y que no constituyen enunciados completos, es decir, las colocaciones.

-Esfera II. Incluye en ella a las unidades fraseológicas que están fijadas en el sistema y que no tienen carácter de enunciado, esto es, las locuciones.

\footnotetext{
${ }^{2}$ La fijación psico-lingüística tiene que ver con la idea de convencionalización y estabilidad en el lexicón de todo hablante de español. En el epígrafe 3 haremos referencia a las propiedades de las UFs.
} 
-Esfera III. Las unidades fraseológicas que se incluyen aquí son las que tienen carácter de enunciado y están fijadas en el habla, esto es, los enunciados fraseológicos ${ }^{3}$.

De este modo, para Corpas, las colocaciones, las locuciones y los enunciados fraseológicos comprenderían la totalidad de UFs que hay en la lengua, al igual que para Ruiz Gurillo (2002a: 453). En los siguientes apartados tratamos las locuciones y los enunciados fraseológicos como elementos de la fraseología. Dejamos fuera a las colocaciones porque, a pesar de que han sido consideradas tanto por Corpas como por Ruiz Gurillo UFs, en nuestra opinión no son elementos de la fraseología, sino de la sintaxis (Alvarado 2008). A continuación, nos centramos en las locuciones y en los enunciados fraseológicos para, posteriormente, ver las diferencias que existen entre ellos.

\subsection{Las locuciones}

Las locuciones han sido estudiadas por diversos autores; sin embargo, nos centramos en Corpas (1996) y Ruiz Gurillo (1997, 2001), puesto que son un punto de referencia en cualquier estudio fraseológico español. Según estas autoras las locuciones son elementos prototípicos de la fraseología. Corpas (1996: 88) define locución como:

UFs del sistema de la lengua con los siguientes rasgos distintivos: fijación interna, unidad de significado y fijación externa pasemática. Estas unidades no constituyen enunciados completos, $\mathrm{y}$, generalmente, funcionan como elementos oracionales.

Además, propone una serie de pruebas formales para comprobar la cohesión semántica y morfológica de las locuciones (Corpas, 1996: 90). Estas pruebas son:

-La sustitución: tiene que ver con la impermutabilidad de un componente de la locución por otro elemento.

-La eliminación: relacionada con la imposibilidad de añadir o sustraer un elemento de la locución.

-Las deficiencias transformativas: imposibilidad de reordenación de sus componentes.

En nuestra opinión, todas las pruebas que presenta Corpas están relacionadas con el criterio de fijación formal que debe tener toda UF, es decir, que estas pruebas se pueden aplicar tanto a las locuciones como a los enunciados fraseológicos. La autora distingue entre locuciones nominales (vacas flacas), adjetivas (sano y salvo), adverbiales (a la vez), verbales (ir y venir), prepositivas (a causa de), conjuntivas (mientras tanto) y clausales (hacérsele a alguien la boca agua), según la función oracional que desempeñen.

Para Ruiz Gurillo las locuciones son sintagmas fijos que, en ciertos casos, presentan idiomaticidad. Según esta autora, las locuciones son los sintagmas más estables del español (Ruiz Gurillo, 2001: 35). Establece dos propuestas de clasificación para las locuciones: una de ellas tiene que ver con sus rasgos internos; otra, con la categoría a la que pertenecen. Así, tendríamos locuciones nominales (empanada mental), adjetivales (corriente y moliente),

\footnotetext{
${ }^{3}$ En ellos se encuentran las fórmulas rutinarias.
} 
verbales (tomar las de Villadiego), adverbiales (al tuntún), marcadoras (sin embargo), prepositivas (en torno a) y clausales (subirsele [a alguien] la sangre a la cabeza). Por tanto, esta clasificación se diferencia de la que ha dado Corpas (1996) por la incorporación de las locuciones marcadoras, que se introducen por su actuación discursiva, ya que la función de los marcadores en el discurso resulta muy importante para la cohesión de un texto, la progresión argumentativa, etc. Este término también ha sido utilizado por autores como Montoro del Arco (2006: 241).

Para nosotros la noción de locución está ligada a la propuesta que ha realizado Ruiz Gurillo (1997, 2001), ya que consideramos que las locuciones son sintagmas fijos de la lengua que se producen y significan en bloque. En ocasiones, ese significado no se corresponde con el significado global de cada una de las partes, esto es, son idiomáticas.

Por tanto, asimilamos tanto la definición como la propuesta de clasificación que hace esta autora al respecto de las locuciones, ya que también cumplen las características que ha de tener toda UF (fijación formal y psico-lingüística ${ }^{4}$ ).

\subsection{Los enunciados fraseológicos}

En este apartado nos dedicamos a estudiar los enunciados fraseológicos, que es donde se encuentran las fórmulas rutinarias, en Corpas (1996), ya que recoge y amplía los presupuestos de Zuluaga (1980), y en Ruiz Gurillo (2000a), puesto que estudia estos enunciados en el español oral. Observaremos que en los enunciados fraseológicos se encuentran tanto paremias como fórmulas rutinarias o citas, es decir, UFs que tienen muy poco en común funcionalmente, lo que nos hace ver que estas UFs no se encuentran en igualdad de condiciones con respecto a otras, en cuanto a estudios y a criterios que las diferencien.

Zuluaga (1980: 191) define los enunciados fraseológicos como expresiones fijas equivalentes o superiores a la frase cuyo "rasgo definitorio es el funcionar como unidades comunicativas mínimas con sentido propio, enunciadas por un hablante entre dos pausas y en unidades de entonación distintas". La clasificación que realiza de este tipo de enunciados se basa en el criterio de fijación e idiomaticidad que poseen.

Corpas (1996) en su Esfera III engloba bajo el nombre de enunciados fraseológicos tanto a paremias como a fórmulas rutinarias. La autora sigue la definición que da Zuluaga (1980: 192) para este tipo de UF:

Las unidades que en nuestro análisis llamamos enunciados fraseológicos funcionan, pues, como secuencias autónomas de habla, su enunciación se lleva a cabo en unidades de entonación distintas; en otras palabras, son unidades de comunicación mínimas.

En esta definición encontramos algunas de las características que deben poseer todo enunciado fraseológico y, por tanto, las fórmulas rutinarias, que se encuentran inmersas en ellos.

\footnotetext{
${ }^{4}$ La fijación debe darse en la forma y en los aspectos psico-lingüísticos, aunque, en ocasiones, también puede aparecer en el nivel semántico-pragmático.
} 
Corpas (1996) diferencia dentro de este tipo de UFs dos subgrupos ${ }^{5}$, las paremias y las fórmulas rutinarias:

a) Las paremias, que tienen autonomía textual y significado referencial, y se dividen a su vez en:

- $\quad$ Enunciados de valor específico, que no poseen valor de verdad general (las paredes oyen o si te he visto no me acuerdo).

- Citas, de origen conocido, proceden de textos o fragmentos hablados de un personaje real o ficticio (la vida es sueño de Calderón o ande yo caliente, y ríase la gente de Góngora).

- $\quad$ Refranes, de origen desconocido (de tal palo, tal astilla).

b) Las fórmulas rutinarias, que carecen de autonomía textual y tienen un significado social, expresivo o discursivo.

- Fórmulas discursivas, que cumplen funciones de organización del discurso (Buenos días, ¿qué tal?).

- $\quad$ Fórmulas psico-sociales, que expresan el estado mental (¡Qué bonito!, ¡madre mía!).

El segundo grupo de enunciados fraseológicos es el que integra nuestro objeto de estudio. Corpas (1996) establece dos tipos de fórmulas rutinarias: discursivas, que cumplen funciones en la organización del discurso, y psico-sociales, que sirven para el desarrollo normal de la interacción social o para expresar los sentimientos de los hablantes. Estas últimas se dividen en diversos subtipos según las distintas funciones pragmático-discursivas que ejercen: expresivas, comisivas, directivas, asertivas, rituales y misceláneas.

Ruiz Gurillo (2000a) define enunciado fraseológico como una combinación fija de palabras con autonomía funcional y, siguiendo la clasificación que propone Corpas (1996), distingue entre paremias y fórmulas rutinarias. Las paremias son estructuras autónomas desde el punto de vista textual, puesto que no vienen determinadas por situaciones y circunstancias concretas; mientras que las fórmulas rutinarias sí están marcadas por una situación social particular.

Por tanto, consideramos que los enunciados fraseológicos se definen como unidades mínimas de comunicación, con independencia, que pueden poseer rasgos internos como la fijación o la idiomaticidad. Dentro de estos enunciados fraseológicos podemos distinguir dos tipos: según tengan significado referencial, las paremias, o significado social, las fórmulas rutinarias. Es aquí donde se encuentra nuestro objeto de estudio que posee unas características concretas, como es la independencia ${ }^{6}$.

\footnotetext{
${ }^{5}$ A medio camino entre las paremias y las fórmulas rutinarias se encuentran los lugares comunes (Corpas, 1996: 150) que pueden presentar un significado denotativo literal, que es lo que coincide con las paremias. Este significado puede ser una verdad aceptada (solo se vive una vez), una cuasitautología (un día es un día) o la expresión de una experiencia (la vida da muchas vueltas). La relación que tienen con las fórmulas rutinarias es de tipo conversacional, ya que se utilizan, por ejemplo, para atenuar la complejidad del acto comunicativo.

${ }^{6}$ Para el análisis de la independencia de las fórmulas rutinarias desde diversos puntos de vista (entonativo, distribucional, textual, semántico y sintáctico, véase Alvarado y Ruiz Gurillo (2008).
} 
Además, debemos destacar en este apartado que, hasta el momento, los enunciados fraseológicos engloban tanto a las paremias como a las fórmulas rutinarias. Este hecho llama la atención si lo comparamos con el apartado anterior en el que las locuciones formaban un único conjunto con homogeneidad en las características funcionales que presentan. Por tanto, bajo ese epígrafe se han englobado estructuras de diferentes características y sólo tienen en común su naturaleza independiente y su ocasional fijación e idiomaticidad $^{7}$. Así pues, tal vez, no se deberían tratar bajo el mismo nombre las paremias y las fórmulas rutinarias, y debería haber un lugar exclusivo en el que se localicen las fórmulas rutinarias y sus rasgos propios. Sin embargo, no es ésta la cuestión que nos ocupa en este momento, sino en centrarnos en su definición.

\section{Las fórmulas rutinarias y sus propiedades}

El término fórmula rutinaria proviene de la etnografía de la comunicación y la etnometodología. Autores como Coulmas (1981b) o Aijmer (1996) se refieren a todo el conjunto de fórmulas como conversational routines, es decir, rutinas conversacionales, de las cuales Coulmas (1981b: 4) afirma que "are tacit agreements which the members of a comunity presume to be shared by every reasonable comember" ${ }^{\prime 8}$. Coulmas lleva a cabo una importante contribución al mundo fraseológico, ya que propone el término de rutina conversacional. Así, a pesar de que no todas las rutinas conversacionales son fórmulas rutinarias, ambas funcionan de acuerdo a sus características en la conversación. De ahí que autores como Corpas se basen en Coulmas para establecer sus denominaciones y realizar su clasificación de UFs.

Corpas (1996: 170) ofrece la siguiente definición para las fórmulas rutinarias:

Se trata de UFS del habla, con carácter de enunciado, las cuales se diferencian de las paremias por carecer de autonomía textual, ya que su aparición viene determinada, en mayor o menor medida, por situaciones comunicativas precisas.

Consideramos que esta definición es muy abarcadora, ya que destaca los rasgos generales de las fórmulas rutinarias, como su carácter social y comunicativo, y olvida otras propiedades importantes, como la idiomaticidad o la fijación. Además, la autora no explica qué entiende por enunciado ni por autonomía textual. El problema que plantea su definición tiene que ver con la vaguedad semántica. De ahí que propongamos una definición, que contenga las características fundamentales para discernir nuestro objeto de estudio.

Las fórmulas rutinarias, como UFs que son, deben poseer las características comunes a todas ellas, la fijación y, en ocasiones, la idiomaticidad, pero además, pueden presentar algún tipo de independencia como enunciados fraseológicos. Consideramos que toda fórmula rutinaria posee fijación formal, entendida como perdurabilidad de los componentes

\footnotetext{
${ }^{7}$ Consideramos que la idiomaticidad es una propiedad semántica posible, pero no fundamental, que se da en las UFs.

8 "Las rutinas conversacionales son acuerdos tácitos que los miembros de una comunidad comparten de forma razonable" (traducción propia).
} 
que la constituyen, y fijación psico-lingüística, referida a la convencionalización en la comunidad lingüística, es decir, a la estabilidad en su reproducción y a su frecuencia de uso. Sin embargo, para las fórmulas rutinarias estos rasgos definitorios se pueden dar de manera gradual. Por ello, establecemos diferentes tipos de fijación y otras propiedades que forman parte de la idiosincrasia de las fórmulas, y que explicaremos seguidamente.

En nuestra opinión, la fijación constituye el rasgo esencial de las UFs en general, ya que gracias a esta propiedad podemos diferenciar si estamos o no ante una UF. Sin embargo, la fijación se puede dar en diferentes niveles y en diferentes grados en las fórmulas rutinarias; de ahí que distingamos entre fijación formal, semántico-pragmática y psico-lingüística. Tomamos estos tres tipos de fijación porque consideramos que la forma y la estabilidad de reproducción y de uso son fundamentales en una fórmula rutinaria, mientras que la fijación semántico-pragmática es una consecuencia posible de las anteriores.

La fijación formal tiene que ver con la fijación interna material a la que hacía alusión Zuluaga (1980) y Corpas (1996). Si aplicamos este concepto a las fórmulas rutinarias, en ellas habría estabilidad en el orden de sus componentes, en sus categorías gramaticales, en su inventario y en su transformatividad. De este modo, las fórmulas rutinarias se conciben como un todo uniforme, capaces de sufrir variantes ${ }^{9}$ sin que conlleven cambio de significado y mantengan su función comunicativa en la conversación. Esta clase de fijación es definitorio de nuestro objeto de estudio y se da en todos los tipos. Veamos el ejemplo (1) en el que varias personas dialogan sobre una de ellas que se ha apuntado al gimnasio:

(1)557 S: [porque ahí entra] la matrículaa/ que son cuatro/ y 558 luego tres mil al mes

559 C: ¡vaya tela! qué mal

$560 \mathrm{~J}$ : pero ¿qué vas a hacer $\uparrow ?$ ¿algún arte marcial o pesas?

(Briz y grupo Val.Es.Co., 2002:156[AP.80.A.1.:557-560])

En (1), la fórmula jvaya tela! presenta fijación formal, ya que hay estabilidad en el orden de sus componentes (*itela va!), en sus categorías gramaticales (*iva tela!) y no permite ni la conmutación (*jvaya ropa!) ni la incorporación de ningún elemento (*jvaya gran tela!). Por todo ello, podemos afirmar que en esta fórmula existe fijación formal.

La fijación semántico-pragmática aparece en las fórmulas rutinarias cuando presentan un significado fijado por el uso que se les da en el contexto habitual en el que se producen. En otras palabras, su valor contextual es el que fija el significado que tiene la fórmula. Si tomamos el ejemplo (1), observamos que la fórmula jvaya tela! tiene fijación semánticopragmática, ya que su significado está fijado en su uso y expresa sorpresa ante la afirmación de A. Esta fórmula sólo puede aparecer en contextos en los que se quiera dar valor expresivo y siempre tiene este tipo de fijación.

\footnotetext{
${ }^{9}$ Consideramos que una fórmula rutinaria presenta variantes cuando presenta un cambio léxico en su estructura, bien sea por adición o reducción, bien por sustitución, sin alterar su significado ni su función en la conversación. Además, incluiríamos dentro de las variantes a las variaciones diafásicas y diatópicas, siempre y cuando sean entendidas por el oyente como tales (Alvarado 2008).
} 
La fijación psico-lingüística es esencial en las fórmulas rutinarias y tiene que ver con los procesos psico-lingüísticos que se relacionan tanto con aspectos de las UFs (institucionalización, convencionalización, etc.), como con los tipos de fórmulas que hay (subjetivas, discursivas, etc.). Se trata de la estabilidad en su reproducción y en la frecuencia de uso que presentan las UFs. Los hablantes las recuerdan y las producen en bloque como una única unidad léxica. En el ejemplo (1) el hablante enuncia la fórmula jvaya tela!, que permanece en su memoria como un todo indisoluble, y es capaz de reproducirla cuando la situación lo permite.

Así pues, este tipo de fijación, aparece siempre junto a la fijación formal, ya que son dos propiedades fundamentales de las UFs y de las fórmulas rutinarias. Sin embargo, la fijación semántico-pragmática no es obligatoria para todas las fórmulas rutinarias. Así, si estamos ante una fórmula cercana al prototipo, presentará los tres tipos de fijación, pero si estamos ante una fórmula periférica tendrá que tener al menos fijación formal y psicolingüística.

La idiomaticidad es una propiedad potencial que puede darse en las fórmulas rutinarias y en las UFs, y consiste en la posibilidad de ausencia de contenido semántico en los elementos que componen la fórmula rutinaria para adquirir un significado que viene dado por su uso en el contexto, es decir, el significado literal desaparece y deja paso al significado figurado.

Por tanto, la fijación y la idiomaticidad se han convertido en las propiedades fundamentales de las UFs; pero en ambos casos se trata de una cuestión de grado. Así, hay UFs totalmente fijas y estables con significado idiomático que constituyen el núcleo de la fraseología (UF prototipo), frente a otras que se repiten en la lengua, pero cuyo grado de fijación es menor y pueden tener un significado literal o ejercer funciones de carácter pragmático. Por su parte, las fórmulas rutinarias poseen también las mismas propiedades, porque forman parte del las UFs. Así, las propiedades de las fórmulas rutinarias son la fijación formal, la fijación psico-lingüística y la independencia, y la potencial idiomaticidad y fijación semántico-pragmática.

Una vez explicadas las propiedades de las fórmulas rutinarias delimitaremos nuestro objeto de estudio con otros tipos de UFs, las locuciones y las paremias.

\section{Las fórmulas rutinarias y las locuciones}

En este apartado intentamos delimitar las fórmulas rutinarias de otras UFs, en este caso de las locuciones. Ya sabemos que tanto las fórmulas rutinarias como las locuciones son UFs y, por tanto, combinaciones formal y psico-lingüísticamente estables de unidades léxicas compuestas por dos o más palabras que forman parte de la competencia léxica de los hablantes, y cuyo límite superior se encuentra en el nivel de la oración compuesta. Además, pueden poseer idiomaticidad.

Las fórmulas rutinarias, además de manifestar esas propiedades, pueden adquirir otras como la independencia, que viene dada por su pertenencia a los enunciados fraseológicos, o la variación, provocada por el uso que hace el hablante de ellas. También debemos tener en cuenta que el significado de las fórmulas, en ocasiones, se extrae del contexto en el que 
éstas se producen o por la función que adquieren en la conversación, es decir, que su significado es contextual o conversacional. Además, pueden tener fuerza ilocutiva, incluso provocar una reacción en el interlocutor, como ocurre con la fórmula jal grano!, que intenta modificar la actuación del oyente. Todas estas características propias de las fórmulas no van a ser compartidas por las locuciones.

Las locuciones, como sintagmas fijos que se producen y significan en bloque, tienen un grado de fijación mayor, con respecto a otras UFs, ya que forman parte del sistema de la lengua y funcionan como elementos sintácticos dentro de las oraciones. Presentan menos grado de independencia que las fórmulas, ya que dependen de la función que realizan en la oración, de la cual depende su significado, es decir, su significado viene dado por la función sintáctica que realizan en la conversación, y del cual depende su taxonomía.

Por tanto, encontramos algunas diferencias en las definiciones de ambas UFs. Las fórmulas rutinarias difieren de las locuciones por su fijación semántico-pragmática y por su potencial independencia, en diferentes grados: entonativa, distribucional, semántica, sintáctica y textual. Sin embargo, pese a estas distinciones, encontramos unidades, como desde luego, que pueden ser tanto locuciones como fórmulas rutinarias. Este hecho se debe a que numerosas expresiones se encuentran a medio camino entre estos dos tipos de UFs por su avanzado estado de pragmatización y convencionalización en sus componentes. Así, desde luego es locución marcadora (Ruiz Gurillo, 2001: 68) cuando forma parte de la sintaxis oracional, es decir, cuando no funciona de forma independiente y aislable en su contexto, como vemos en el ejemplo (2), mientras que es fórmula rutinaria y funciona como evidencial, cuando su significado viene dado por el contexto en el que se produce y es además aislable dentro de él, esto es, no forma parte de la sintaxis oracional, como vemos en el ejemplo (3). En (2) dos matrimonios cuentan las anécdotas de un viaje que hicieron a Galicia:

(2) J: una parada de esas quee/// eso es aparte// está con su hija $\uparrow / /$ y come aparte $\uparrow /$ o se va a cagar aparte// porque está hablando cosas de familia y eso/ eso desde luego no lo veo $\mathrm{mal} / / /$ entramos ahí en un bar $\uparrow / /$ una de esas paradas que se baja todo el mundo a tomarse un café o tomarse una/// unas cervezas o algo/ o lo que sea $\downarrow$ claro todo el mundo (( ))

(Briz y grupo Val.Es.Co., 2002: 278[PG.119.A.1:90-93])

En (3) varios interlocutores hablan sobre un reloj de gran valor que se han encontrado en la calle: 


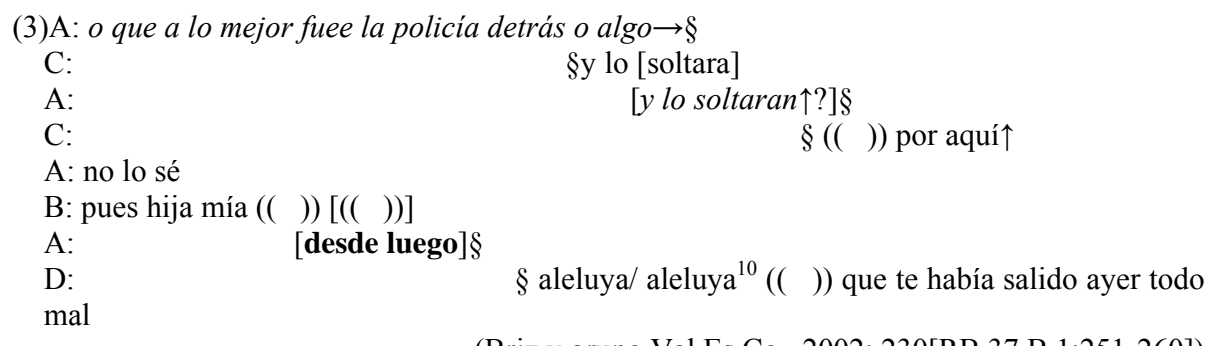

En (2) desde luego forma parte del cuerpo del texto, es decir, funciona como un operador e intensifica la opinión del hablante. Así, desde luego no es totalmente independiente del contexto en el que se produce y no tiene significado por sí misma, es decir, no posee valor formulístico en la conversación, mientras que en (3) se trata de una fórmula que se puede desligar de su sintaxis, es independiente y sirve para mostrar acuerdo y certeza, y funciona como un evidencial. De este modo, la independencia en alguno de sus aspectos y el valor que poseen en la conversación son los rasgos que nos permiten distinguir entre estas dos UFs.

Por tanto, la distinción entre locuciones y fórmulas rutinarias tiene que ver, aparte de las características propias de cada una de ellas, con la función que realizan en el contexto en el que se dan.

Una vez que hemos diferenciado las fórmulas rutinarias de las locuciones, veremos, a continuación, la frontera de nuestras unidades objeto de estudio con otro tipo de enunciado fraseológico, las paremias.

\section{Las fórmulas rutinarias y las paremias}

Ya sabemos que ambos tipos de UFs forman parte de los enunciados fraseológicos, compuestos por fórmulas rutinarias y paremias, que se definen como unidades mínimas de comunicación que pueden poseer rasgos internos como la fijación o la idiomaticidad. Corpas (1996: 170) se pronuncia al respecto y diferencia las fórmulas rutinarias de las paremias por "carecer de autonomía textual, ya que su aparición viene determinada en mayor o menor medida, por situaciones comunicativas precisas", es decir, dependen del contexto en el que se producen y su significado es discursivo, social o expresivo.

Por tanto, a nuestro modo de ver, la distinción entre las unidades que componen los enunciados fraseológicos tiene que ver con el significado que presentan. Así, las paremias tienen un significado referencial, es decir, su significado se relaciona con una verdad general, es transparente en la mayoría de los casos y, normalmente, va dirigido como consejo al oyente, como asi es la vida o para presumir hay que sufrir; pero también puede ser figurado como quien a buen árbol se arrima, buena sombra le cobija. Mientras que las fórmulas rutinarias poseen un significado social o discursivo y muestran la actitud del

${ }^{10}$ Cantando. 
hablante ante el enunciado, como observamos en el ejemplo (4) en el que varios amigos hablan sobre cómo son los curas y sobre la cena de clase que tuvo uno de ellos:

(4) E: yo es que personalmente no conozco a ninguno/ yo conozco a (( )) de vista// un día que fui// pero hace poco tuve una cena $\uparrow$ hizo una- una cena de- de universidad// y bueno pues/ no sé (( ) )/// y la gente una pinta toda/ conn elll traje chaqueta/ y yo iba con los vaqueros/ hecha polvo /todo el mundo allí puesto ¿no? ¡madre mía! ¡qué asco! (...)

(Briz y grupo Val.Es.Co., 2002:84[L.15.A.2.:93-104])

En el ejemplo (4) el hablante enuncia la fórmula ;madre mía! para expresar sorpresa ante la vestimenta que llevaban sus compañeros en una cena de universidad, es decir, se utiliza para mostrar la subjetividad del hablante en el enunciado.

Por tanto, la confusión entre paremias y fórmulas rutinarias se disuelve si nos atenemos al tipo de significado y función que presentan.

\section{Conclusiones}

En este trabajo hemos analizado los rasgos que caracterizan a las fórmulas rutinarias como unidades que pertenecen al ámbito de la fraseología. Si difícil ha sido para los investigadores la elección de un término que denomine nuestro objeto de estudio, no menos complicado ha sido recopilar las propiedades que poseen, como hemos hecho en el epígrafe 3. Así pues, hemos distinguido las UFs que engloban esta disciplina y las diferencias que existen entre unas unidades y otras. Por eso, no sólo nos hemos atrevido a dar una definición de fórmula rutinaria, entendida como UF independiente, que está compuesta por dos o más elementos fijados en la forma y convencionalizados en la comunidad de habla, y puede presentar idiomaticidad, a veces causada por la fijación semántica; sino que también hemos intentado delimitarlas de otras UFs, como las locuciones o las paremias, con el fin de esclarecer estas unidades tan comunes en la lengua hablada. Sin embargo, seguimos observando problemas en el grupo de los enunciados fraseológicos, ya que se insertan en ellos unidades de diversa índole, por lo que habría que replantear un nuevo estudio.

A modo de conclusión podemos afirmar que la diferencia que existe entre las fórmulas rutinarias y las locuciones tiene que ver con el grado de fijación y la independencia que tienen, es decir, con sus rasgos, mientras que la diferencia que existe entre las fórmulas rutinarias y las paremias tiene que ver con el tipo de significado que poseen.

\section{Referencias bibliográficas}

Aijmer, K. (1996): Conversational Routines in English. London, Longman.

Alvarado Ortega, M. B. (2006): “¿Son las fórmulas rutinarias enunciados independientes?”. En Face, T. L. y C. A. Klee (eds.): Selected Proceedings of the 8th Hispanic Linguistics Symposium. Somerville, Cascadilla Proceedings Project, págs. 214-220. 
Alvarado Ortega, M. B. y L. Ruiz Gurillo (2008): "Unidades de la conversación y fraseología: acerca de la autonomía de las fórmulas rutinarias". En Aspectos formales y discursivos de las expresiones fijas. Frankfurt a. M./ Berlin, Peter Lang, págs. 25-40.

Alvarado Ortega, M. B. (2008): Las fórmulas rutinarias en el español actual. (Tesis doctoral inédita)

Azorín Fernández, D. (Coord.) (2002): ALCORE. Alicante Corpus del Español. ISBN: 84-7908-684$\mathrm{X}$.

Briz, A. y Grupo Val.Es.Co. (2002): Corpus de conversaciones coloquiales. Madrid, Arco Libros.

Burger, H. (2000): "El concepto de variación en fraseología". En A. Pamies y J. D. Luque (eds.): Trabajos de lexicografía y fraseología contrastiva. Granada, Método ediciones, págs. 105-117.

Casares, J. ([1950] 1969): Introducción a la lexicografía moderna. Madrid, Revista de Filología Española, Anejo LII.

Corpas, G. (1996): Manual de fraseología española. Madrid, Gredos.

Coulmas, F. (1979): "On the sociolinguistic relevante of routine formulae". En Journal of Pragmatics, 3, págs. 239-266.

Coulmas, F. (1981a): "Idiomaticity as a problem of pragmatics". En H. Parret, M. Sbisà y J. Verschueren (eds.): Possibilities and limitations of pragmatics: Procedings of the Conference on Pragmatics, Urbino, July 8-14, 1979. Amsterdan, John Benjamins, págs. 139-151.

Coulmas, F. (ed.) (1981b): Conversational Routine. Explorations in Standardized Communication Situations and Prepatterned Speech. Vol. II, La Haya, Mouton.

Hernando L. A. (1990): "Sobre las unidades fraseológicas en español". En Actas de la Sociedad Española de Lingüística. XX Aniversario, Vol. 1, págs. 538-546.

Montoro del Arco, E. T. (2006): Teoría fraseológica de las locuciones particulares. Las locuciones prepositivas, conjuntivas y marcadoras en español. Frankfurt am Main, Peter Lang.

Ruiz Gurillo, L. (1996): "Sobre la fraseología coloquial: corpus e investigación”. En Actas del I Congreso Internacional de AESLA (Granada, septiembre de 1992), págs. 493-498.

Ruiz Gurillo, L. (1997): Aspectos de fraseología teórica española. Valencia, Universidad, Anejo XXIV de Cuadernos de Filología.

Ruiz Gurillo, L. (1998): La fraseología del español coloquial. Barcelona, Ariel.

Ruiz Gurillo, L. (2000a): "La fraseología”. En Briz, A. y Grupo Val.Es.Co. (coords.): ¿Cómo se comenta un texto coloquial? Barcelona, Ariel, págs. 169-189.

Ruiz Gurillo, L. (2000b): “Puede ser la fraseología relevante en cualquier situación?”. En Salvador, V. y A. Piquer (eds.): El discurs prefabricat. Estudis de fraseología teórica i aplicada. Actes de les Jornades de Fraseología Comparada. (Castellón, noviembre de 1999). Castellón, Universidad, págs. 81-103.

Ruiz Gurillo, L. (2001): Las locuciones en español actual. Madrid, Arco Libros

Ruiz Gurillo, L. (2002a): "La neología nominal sintagmática en el español técnico". En A. Bernabé; J.A. Berenguer; M. Cantanero y J. C. Torres (eds.): Presente y futuro de la Lingüística en España: La sociedad de lingüística 30 años después, II, págs. 449-457.

Ruiz Gurillo, L. (2002b): "Compuestos, colocaciones, locuciones: intento de delimitación". En A. Veiga; M. González y M. Souto (eds.): Lenguas e Lingüistica: Léxico y Gramática, 3. Lugo, Tris tram, págs. 325-339.

Zamora, P. (1998): "Las frases idiomáticas pragmáticas italianas: los marcos de situación y sus equivalencias en español”. En Estudios románicos. 10, págs. 107-125.

Zamora, P. (1999): "Otro intento de clasificación y delimitación de unidades fraseológicas italianas". En Homenaje al profesor Trigueros Cano. II, Murcia, págs. 733-750.

Zuluaga, A. (1980): Introducción al estudio de las expresiones fijas. Tübingen, Max Hueber, Verlag. (Tesis doctoral inédita). 
Zuluaga, A. (1992): "Spanisch: Phraseologie". En Holtus, G; M. Metzeltin y CH. Schmitt (eds.): Lexikon der Romanistischen Linguistik. Tübingen, Max Niemeyer, Verlag, págs.125-131. 